\title{
Cervix remodeling and parturition in the rat: lack of a role for hypogastric innervation
}

\author{
Jonathan W Boyd ${ }^{3}$, Thomas J Lechuga ${ }^{3}$, Charlotte A Ebner ${ }^{3}$, Michael A Kirby ${ }^{1,2,3}$ \\ and Steven M Yellon ${ }^{1,2,3}$ \\ Departments of ${ }^{1}$ Physiology, Pathology and Human Anatomy and ${ }^{2}$ Pediatrics and ${ }^{3}$ Center for Perinatal Biology, Loma \\ Linda University School of Medicine, Loma Linda, California 92350, USA
}

Correspondence should be addressed to S M Yellon; Email: syellon@Ilu.edu

\begin{abstract}
The hypogastric nerve is a major pathway innervating the uterine cervix, yet its contribution to the processes of cervical ripening and parturition is not known. The main objective of this study was to determine the effect of hypogastric nerve transection on remodeling of the cervix and timing of birth. As an initial goal, processes associated with remodeling of the peripartum cervix were studied. The cervix was obtained from time-dated pregnant rats on days 15, 19, 21, and 21.5 of pregnancy, and post partum on the day of birth (day 22). The cervix was excised, post-fixed overnight, and sections stained to evaluate collagen content and structure or processed by immunohistochemistry to identify macrophages or nerve fibers. The census of macrophages and density of nerve fibers in the cervix peaked on day 21, the day before birth, and then declined post partum. These results replicate in time course and magnitude previous studies in mice. To address the main objective, the hypogastric nerve was bilaterally transected on day 15 post-breeding; sham-operated rats served as controls. Pups were born in both groups at normal term. Transection of the hypogastric nerves did not affect remodeling of collagen or the census of macrophages or the density of nerve fibers in the cervix. These findings support the contention that enhanced innervation and immigration of immune cells are associated with remodeling of the cervix and parturition, but that a neural pathway other than the hypogastric nerve may participate in the process of cervical ripening.

Reproduction (2009) 137 739-748
\end{abstract}

\section{Introduction}

The cervix functions as a gatekeeper to protect the developing fetus, as well as contents of the pregnant uterus, from external vaginal ecology. After an appropriate duration of pregnancy, the cervix undergoes a remodeling process and birth occurs at a precise time of day in rodents and, to an extent, in humans (Longo \& Yellon 1988). Recent findings indicate that the density of nerve fibers in the cervix is increased by the day before birth in mice (Kirby et al. 2005, Yellon et al. 2008). Since ripening of the cervix resembles a neuroimmune-mediated inflammatory response (Mackler et al. 1999, Richardson \& Vasko 2002, Yellon et al. 2003), our attention has focused on the role that nerves may serve in processes associated with remodeling of the cervix at the conclusion of pregnancy.

The lower reproductive tract in females is highly innervated in non-pregnant (NP) humans and rodents. Innervation of the uterine cervix involves a topography of nerve fibers that, depending upon species, project from the lower thoracic, lumbar, and upper sacral spinal cord (Papka et al. 1987, Berkley et al. 1993, Sullivan et al. 1994, Houdeau et al. 1998). In the rat, spinal cord segments from thoracic 13 to lumbar 3 have afferent connections via the hypogastric nerve and inferior mesenteric ganglion to caudal portions of the uterine horns and the uterine cervix (Baljet \& Drukker 1980, Peters et al. 1987, Lee \& Erskine 2000). The hypogastric nerve makes a significant contribution to the total innervation of the cervix in NP females (Owman 1981, Steinman et al. 1992). Hypogastric nerve fibers in the rat cervix are reported to regulate neurogenic motor responses of smooth muscle (Owman 1981, Stjernquist \& Owman 1987, Papka \& Traurig 1988, Melo \& Machado 1993) and are components of a sensory afferent pathway that mediates nociception (Cunningham et al. 1991, Sandner-Kiesling et al. 2002). Transection of the hypogastric nerve is found to attenuate the increase in pain threshold during pregnancy (Gintzler et al. 1983). Moreover, hypogastric neurectomy abolishes behavioral response to uterine horn distention (Temple et al. 1999) and is reported to eliminate the perception of intense mechanical stimulation of the uterine horns (Berkley et al. 1993). In contrast to evidence that suggests a role for the pelvic nerve in receptivity to vaginocervical stimulation, ovulation, and the process of parturition (Carlson \& De Feo 1965, Martinez-Gomez et al. 1998), 
little is known about the importance of the hypogastric nerve in pregnancy or its contribution to processes associated with remodeling of the cervix and the process of birth. Thus, the dual focus of the present study was to establish the time course of characteristics for cervical ripening associated with parturition in the rat and to test the hypothesis that transection of the hypogastric nerve eliminates a critical pathway that regulates remodeling of the cervix and timing of birth.

\section{Results}

\section{Experiment 1: remodeling in the peripartum cervix}

As pregnancy neared term, hypertrophy was evident in the thickness of the luminal epithelium and reduced the numbers of cell nuclei per volume of tissue. More space surrounded stromal cells and nuclei, while smooth muscle cells appeared elongated in pregnant compared with NP cervices. Mean numbers of cell nuclei $( \pm$ S.E.M. $) / \mu \mathrm{m}^{3} \times 10^{4}$ were for NP: $11.07 \pm 0.26$, D15:
8.24 \pm 0.38, D19: $6.87 \pm 0.38$, D21: $6.17 \pm 0.35$, D21.5: $5.51 \pm 0.37$, and PP: $6.61 \pm 0.42$ respectively $(P<0.05$, ANOVA $\mathrm{F}=45.22, \mathrm{df}=5$; cell nuclei density for $\mathrm{NP}$ controls > all other groups; D15 > D19, D21, D21.5, and PP groups; and D19>D21.5 group). Based upon the morphology of cells in the cervix and vaginal epithelium, NP rats were in the diestrus phase of their reproductive cycle. Thus, compared with NP controls, cells in stromal and smooth muscle areas of cervix appeared to undergo a twofold increase in size with pregnancy and, during the last 6 days before birth, further hypertrophy by more than $30 \%$.

Collagen structure in the cervix varied with respect to reproductive status. Bright red stained collagen fibers were densely packed and regularly arranged in the smooth muscle dense region between luminal epithelium and stroma in NP rat cervix (Fig. 1). On day 15 of pregnancy, collagen structure was well organized but thinner and less dense compared with those in NP cervix. By day 19 of pregnancy, the density of birefringence was markedly reduced; spaces were evident
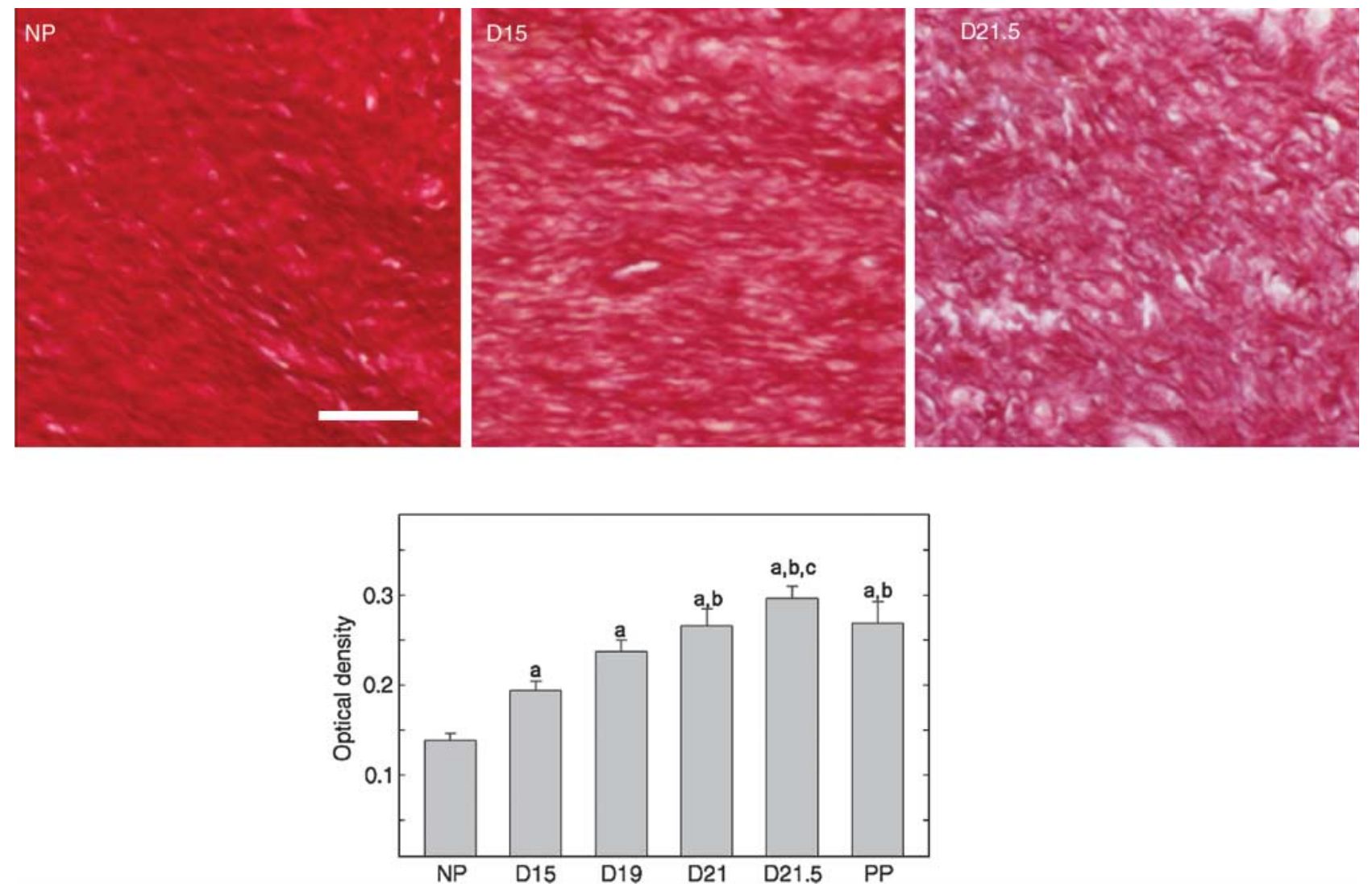

Figure 1 Top panels. Photomicrographs of picrosirius-stained collagen in the cervix from rats that were non-pregnant (NP), day 15 pregnant (D15, 7 days before birth) or prepartum (D21.5, day before birth). Bright birefringence was evident as intense orange on a light yellow background. Scale bar $=50 \mu \mathrm{m}$. Bottom panel. Data are the mean optical density of birefringence from picrosirius red-stained sections of cervix (OD) \pm S.E.M. from pregnant rats on day 15, 19, 21 or 21.5 post-breeding (D15, D19, D21 or D21.5) and post partum (PP; day 22 post-breeding) and were normalized to total cell nuclei density in NP rats ( $n=3$ sections from each of 5-6 rats per group). Color photomicrographs were converted to grayscale images; regions of dense collagen appear as dark areas, i.e., high birefringence, while whiter areas represent reduced collagen and scattered fibrils (details in Materials and Methods). Thus, higher OD values represent reduced birefringence, indicative of reduced collagen and diffuse structure. Letter symbols indicate $P<0.05$ : 'a' versus $N P$, ' $b$ ' versus D15, and ' $c$ ' versus D19 $(P<0.05$; ANOVA; $F=20.48, d f=5)$. 
between fibrils. After birth (day 22 post-breeding), this pale red stain, an indication of sparse collagen or lack of structure, was intermixed with areas of more aligned and closely packed collagen. Optical density reflected these changes in birefringence of polarized light from sections of peripartum cervix. Typical of that found in cervix from NP mice, optical density measures were low when birefringence was high, an indication of dense collagen content and complex structure. As corrected for hypertrophy of tissue with pregnancy, mean optical density increased by day 15 of pregnancy relative to that in NP controls $(P<0.05$, ANOVA). The peak optical density by day 21.5 of pregnancy indicated that collagen content and structure was maximally altered by the day before birth. In postpartum rats, optical density remained increased relative to that in cervices from NP or day 15 pregnant rats. Thus, collagen structure had yet to return to that found in un-ripened cervix.

The morphology and anatomical distribution of ED-1stained macrophages was comparable with that in previous reports of macrophages that reside in the murine cervix. ED-1-stained cells were evident in the stroma, around blood vessels, and in the submucosal epithelium (Fig. 2). Dark-stained cells were associated with counterstained cell nuclei. More macrophages were present in stroma and smooth muscle regions of the peripartum cervix compared with that earlier in pregnancy or in NP rats. Macrophage numbers in the cervix varied with respect to stage of pregnancy. The peak number of stained cells occurred on day 21.5 of pregnancy and remained elevated in the postpartum group compared with that in rats on day 15 of pregnancy or in NP controls.

Nerve fibers, as identified by an antibody to neurofilament protein peripherin, were stained brown and morphologically punctate, likely fibers in cross-section, or extended lengths of thin individual or interwoven fibrils. Occasionally, fibers had beaded varicosities. Nerve fibers were predominantly found within the subepithelial stroma, interspersed between smooth muscle bundles in the perimetrium, and sometimes associated with blood vessels (Fig. 3). With hypertrophy of cervix during pregnancy, nerve fibers were increasingly present in stroma and regions of smooth muscle. The area of cervix that contained nerve fibers was significantly increased by day 15 of pregnancy compared
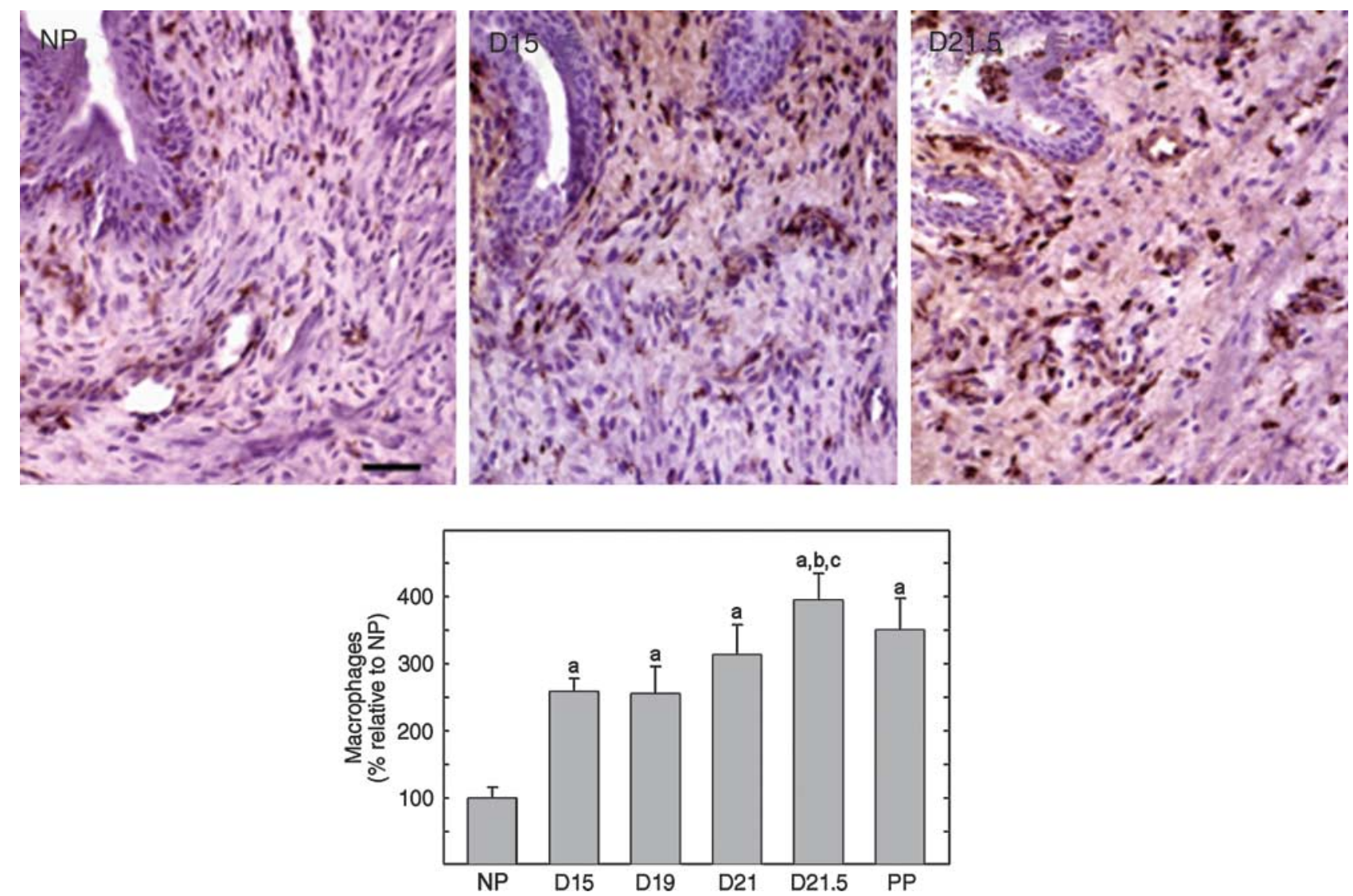

Figure 2 Top panels. Photomicrographs of ED-1-stained macrophages in the rat cervix. Macrophages were darkly stained and sections counterstained with hematoxylin (under the microscope, cells were brown with violet counterstain of nuclei). Scale bar $=50 \mu \mathrm{m}$. Bottom panel. Mean number of macrophages ( \pm S.E.M.; $n=5-6$ rats/group) in the cervix of rats normalized to cell nuclei relative to that in the non-pregnant group. Mean numbers of macrophages in NP rats were $3.30 \mathrm{cells} / \mu \mathrm{m}^{3} \times 10^{2}$ nucleus area in cervix. See Materials and Methods for details of the cellcounting procedure. Group designations and letter symbols of statistical analysis are the same as in Fig. $1(P<0.05 ; \mathrm{ANOVA} ; F=9.39, \mathrm{df}=5)$. 

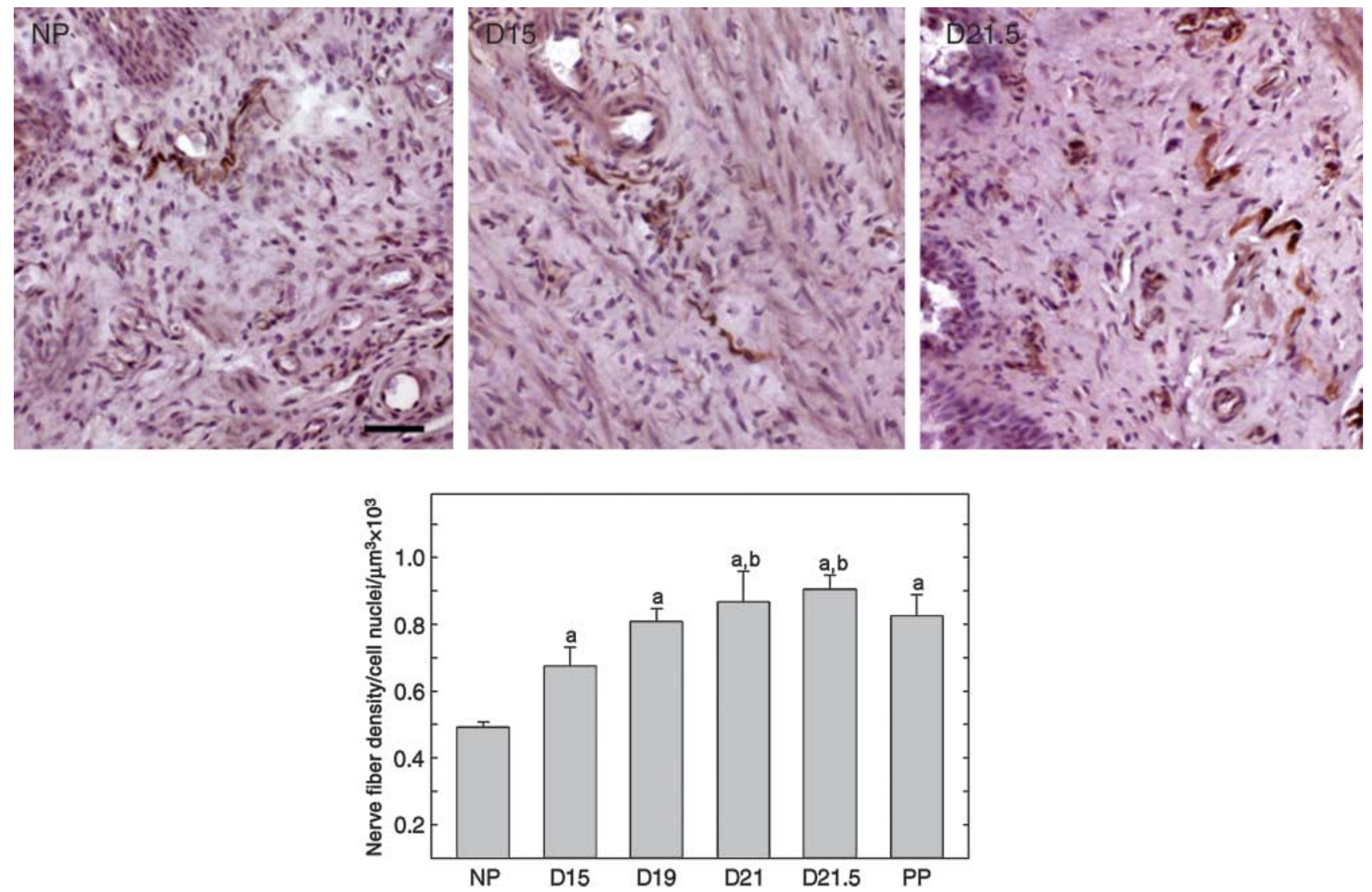

Figure 3 Top panels. Photomicrographs of peripherin-stained nerve fibers in the rat cervix. Nerve fibers were darkly stained and sections were counterstained with hematoxylin (under the microscope, fibers were brown with violet counterstain of nuclei). Scale bar $=50 \mu \mathrm{m}$. Bottom panel. Under brightfield microscopy, boxes in an eyepiece reticle grid ( $10 \times 10$ boxes) that contained immunoreactive nerve fibers were counted, multiplied by the area of the grid $\left(2401 \mu \mathrm{m}^{2} /\right.$ box $\times 10 \mu \mathrm{m}$ section thickness), then normalized to average cell nuclei counts in the respective group and to cell nuclei counts in non-pregnant rats to account for hypertrophy of cervix with pregnancy and for variability related to individual tissue sections. Data are the mean fiber density/area of cervix ( \pm S.E.M.; $n=5-6$ rats/group) in at least 21 non-overlapping vertical and horizontal grid placements in a total of two sections from each cervix. Group designations and letter symbols of statistical analysis are the same as in Fig. $1(P<0.05 ; \mathrm{ANOVA} ; F=8.74, \mathrm{df}=5)$.

with that in NP rats. Further increases in the density of nerve fibers were evident by day 19 of pregnancy with peak area of innervation found by the day before birth, day 21.5 of pregnancy. After birth, the density and distribution of nerve fibers was not significantly different from values on days 15 and 19 of pregnancy.

\section{Experiment 2: effect of $\mathrm{HnX}$ on timing of birth}

In Sham-operated controls, births occurred spontaneously at normal term (Table 1). The timing of birth was not significantly different in rats with hypogastric nerve transections. Most rats gave birth on day 22 of pregnancy; one of $6 \mathrm{HnX}$ rats gave birth on day 23 postbreeding. Pups in both groups were found alive in the morning on the day of birth. Thus, transection of the hypogastric nerve did not interfere with the timing of birth or delivery of pups. Moreover, transection of the hypogastric nerve did not affect hypertrophy of the cervix with pregnancy. The cell nuclei per area of cervix was equivalent in groups of Sham and HnX rats, as well as to the cell nuclei density in PP rats in Experiment 1 ( $P>0.05$, Student's $t$-test).

Transection of the hypogastric nerves did not affect remodeling of the cervix at the conclusion of pregnancy. Birefringence of polarized light from picrosirius red-stained sections was similar in Sham and $\mathrm{HnX}$ rats; comparable with that seen in unoperated PP rats in Experiment 1. Collagen fibers were loosely and diffusely distributed in sections of cervix from both groups. Open pockets with sparse picrosirius red stain were evident in portions of most sections irrespective of treatment.

Table 1 Day of birth, pups/litter, and density of cell nuclei in the cervix of sham-operated controls (Sham) or hypogastric nerve transected rats $(\mathrm{HnX})$.

\begin{tabular}{lllcc}
\hline Group & $\boldsymbol{n}$ & Day of birth & Pups/litter & $\begin{array}{c}\text { Cell nuclei } \\
\left(\# / \mu \mathrm{m}^{3} \times 10^{-4}\right)\end{array}$ \\
\hline Sham & 5 & 22 & $10 \pm 3.4$ & $4.15 \pm 0.7$ \\
HnX & 6 & $22.2 \pm 0.4$ & $9 \pm 3.6$ & $4.09 \pm 1.4$ \\
\hline
\end{tabular}

Data are mean \pm S.E.M.; $n=$ number of rats/group. 

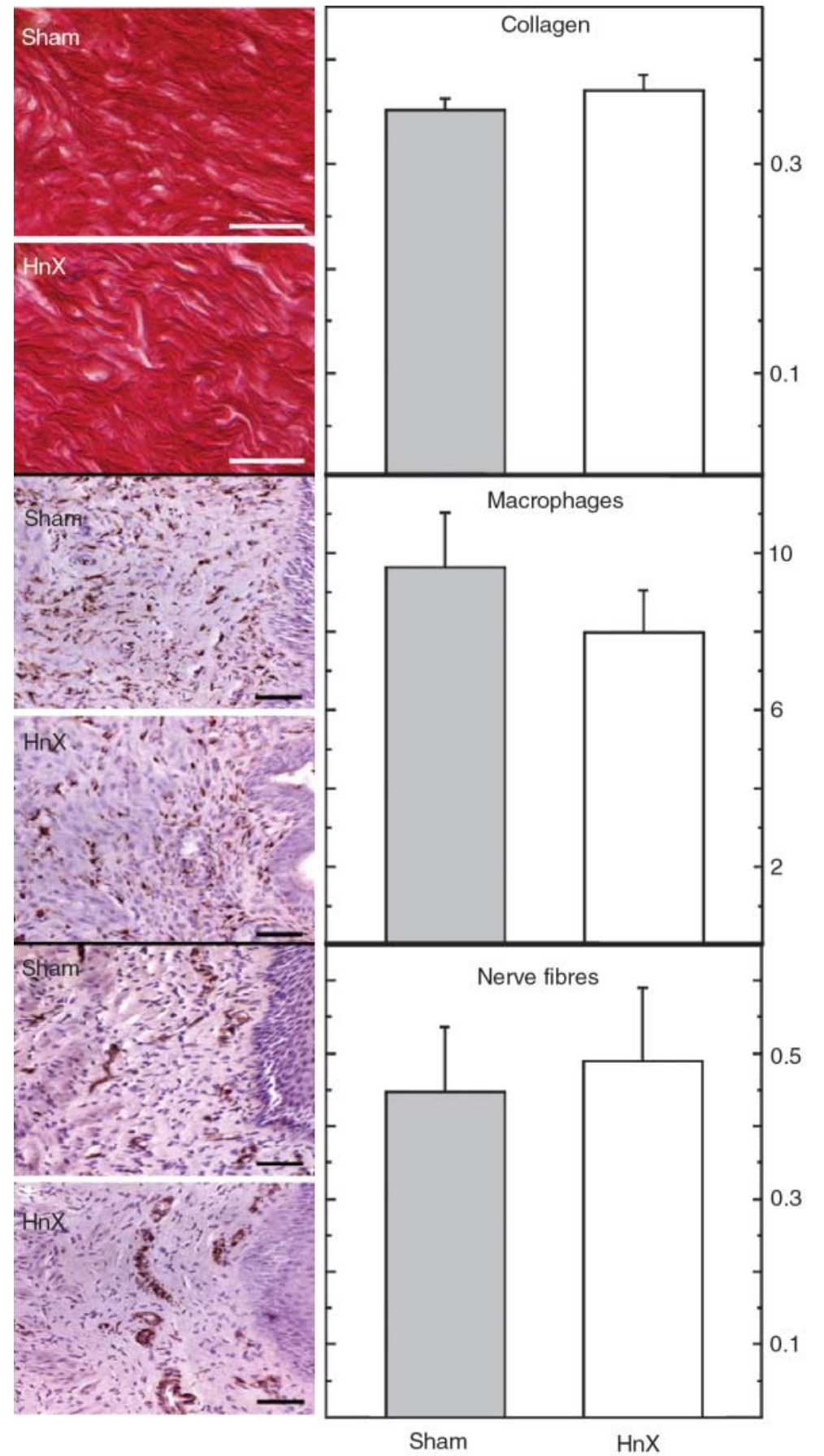

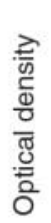

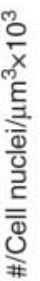

Figure 4 Top panels. Photomicrographs of collagen fibers in the cervix of rats that were sham-operated (Sham) or had their hypogastric nerve transected $(\mathrm{HnX})$ on day $14-15$ of pregnancy. Graph indicates the mean optical density ( \pm s.E.M.; 3 sections/rat, $n=5-6$ rats in each group) of birefringence of polarized light normalized to cell nuclei density to account for variability due to hypertrophy of cervix with pregnancy, as well as among individual sections and mice. $P>0.05 \mathrm{HnX}$ versus Sham, Student's $t$-test, $F=3.11$ ). Middle panels. Photomicrographs of macrophages in the cervix of a Sham or HnX rat. Graph is the mean number of macrophages normalized to total cell nuclei density in cervix of each group ( \pm S.E.M., $n=5-6 ; P>0.05$, Student's $t$-test, $F=0.06$ ). Bottom panels. Photomicro-graphs of nerve fibers in the cervix of a Sham or HnX rat. Note spiral of thick nerve fiber bundle. Mean area \pm s.E.M. of cervical tissue with nerve fibers normalized to total cell nuclei density ( $n=5-6$ rats; $P>0.05$, Student's $t$-test, $F=0.04)$. Scale bars $=50 \mu \mathrm{m}$.
Optical density was equivalent between Sham and $\mathrm{HnX}$ groups $(P=0.11, \mathrm{df}=9$, Student's $t$-test; Fig. 4 top). In addition, the census of dark brown- ED-1-stained macrophages was not significantly different between Sham and HnX groups $(P=0.99, \mathrm{~d} f=9$, Student's $t$-test; Fig. 4 middle). Finally, peripherin-stained nerve fibers were commonly found near blood vessels and as bundles in subepithelial regions of the cervix. The area of cervix that contained nerve fibers was the same in Sham controls and $\mathrm{HnX}$ rats $(P=0.86, \mathrm{df}=9$,
Student's t-test; Fig. 4 bottom). Thus, collagen content and complexity of structure, as well as density of macrophages and innervation were similar in Sham and $\mathrm{HnX}$ rats.

\section{Discussion}

The present findings indicate that hypertrophy of cervix, reduced collagen content and structure, enhanced presence of immune cells, and increased local 
innervation are processes that characterize prepartum remodeling of the cervix in the rat. These findings correlate well with previous reports of parameters associated with cervical remodeling in several strains of mice (Mackler et al. 1999, Kirby et al. 2005, Yellon et al. 2008), as well as to replicate the time course for prepartum changes in collagen structure (Marx et al. 2006) using a different method of analysis, optical density versus luminosity. Consistent with (Bosquiazzo et al. 2005), the census of macrophages in the cervix peaked by the day before birth, $12 \mathrm{~h}$ or so before pups delivered in the present study. Collectively, the evidence supports the proposition that remodeling of the cervix involves an increased presence of macrophages and nerve fibers during the peripartum period. Recruitment of macrophages may be important for the remodeling process because these immune cells produce proinflammatory cytokines, contain leukocyte collagenases, and are a source for prostaglandins and nitric oxide (Junqueira et al. 1980, Hertelendy \& Zakar 2004, Doherty \& Broide 2007). Each of these factors promotes inflammatory processes that include collagen breakdown, edema, and vasodilation in other tissues. In addition, evidence in rabbits (Uchiyama et al. 1992) and women (Tanaka et al. 1998, Facchinetti et al. 2005, Huber et al. 2005, Tornblom et al. 2005) indicates that proinflammatory cytokines, i.e., message and/or protein concentrations, are increased in association with ripening of the cervix at term. The temporal correlation between increased immune cell-associated products and enhanced numbers of macrophages raise the possibility that immune cells may participate in processes of relaxation, remodeling, and ripening of the cervix in the preparation for parturition. These findings have implications in humans for similar processes associated with ripening of the cervix (Ekman-Ordeberg et al. 2003).

The importance of a peak in nerve fibers in the prepartum cervix has yet to be determined. After day 19 , the distribution of nerve fibers during the period leading up to birth was relatively stable and did not keep pace with the significant hypertrophy of cells in the cervix. Neuropeptidergic nerve fibers, such as those that stain for calcitonin gene-related peptide (CGRP), densely innervate the rat cervix (Mowa et al. 2003) and, in the mouse, the density of CGRP-immunoreactive fibers increase by the day before birth (Kirby et al. 2005). Other neuropeptidergic fibers that are present within the cervix (Traurig et al. 1991, Papka \& McNeill 1993) could also serve neuroeffector functions of sensory fibers to promote vasodilation or leukocyte immigration (Holzer 1988). The idea that neural secretions may mediate local immigration or activation of immune cells extends from studies in the cutaneous immune system where sensory neuropeptides promote inflammation (Collins et al. 1998, Kong et al. 1998, Richardson \& Vasko 2002). Thus, neurogenic activation of an inflammatory immune reaction in the cervix may be a crucial upstream component of the multiphasic mechanism described by Challis et al. (2002) for the transition from Phase 0 to 1 in the process of parturition.

Findings that support an increase in immune cells in the prepartum rodent cervix are based upon studies in which specific immunohistochemically stained cells were counted in a known volume of tissue (Mackler et al. 1999, Yellon et al. 2008). Although the cervix increases in mass with pregnancy compared with that when not pregnant, i.e., hypertrophy of the cervix reflects an increase in total number of cells and extracellular space, the actual numbers of cell nuclei per area of a microscopic field significantly decreases by the day before birth versus day 15 of pregnancy in rodents or compared with that in NP controls. As stroma and smooth muscle cells in the cervix increase in size with pregnancy, evident in photomicrographs in the present study and other reports (Mackler et al. 1999, Timmons \& Mahendroo 2006, Yellon et al. 2008), intercellular spaces decrease in area of microscopic analysis. Even though the area within the extracellular matrix for immune cells to occupy was reduced, the findings in rodents, our past studies in mice and by Bosquiazzo et al. in Wistar rats, indicate that the census of immune cells in the cervix increase several fold relative to the total population of cells in the cervix. Greater numbers of immune cells are likely due to immigration into the peripartum cervix, since macrophages and neutrophils differentiate, but do not replicate in tissue. In mice and rats, the time course for immigration of macrophages is remarkably similar; peak numbers occur by the day before birth and remain elevated on the day of birth. This finding is consistent with evidence that leukocytes are increased in the cervix from women at term relative to that earlier in pregnancy (Bokstrom et al. 1997). Whether this increase occurs before or after the start of labor is controversial (Osman et al. 2003) and may depend upon the complex challenge to obtain cervical biopsies from non-laboring women at term.

To address the second objective of the present study, the findings support the contention that the hypogastric nerve does not regulate cervical ripening or the process of parturition. In the peripartum cervix, collagen content and structure, macrophage census, and density of nerve fibers were the same as in rats that had bilateral transection of the hypogastric nerve as in sham-operated controls. These results raise the possibility that a neural pathway other than that involving the hypogastric nerve may affect cervical ripening. Transection of the pelvic nerve, the sensory viscerocutaneous branch in particular, is associated with delayed onset of birth, dystocia, prolonged duration of labor or prevention of delivery in a majority of rats (Burden et al. 1990, Martinez-Gomez et al. 1998). With the exception that collagen density was observed to increase in the cervix after pelvic neurectomy (Burden et al. 1984), the consequences of 
pelvic nerve transection on morphological processes associated with ripening of the cervix are not known. Moreover, based upon evidence in NP rats, innervation by the vagus nerve may also be important to regulate cervical function (Cueva-Rolon et al. 1996, Collins et al. 1999, Guevara-Guzman et al. 2001). In conjunction with the lack of effect of hypogastric nerve transection on the timing of birth, the data clearly indicate that innervation of the lower uterus and cervix by the hypogastric nerve is unlikely to have a role in the process of parturition at normal term.

Other important physiological functions are mediated by the hypogastric innervation of the lower uterus and cervix. This nerve projection is reported to be involved in micturition, blood flow, and uterine contractility (de Groat \& Theobald 1976, Sato et al. 1989, 1996, Vera et al. 1997, Dmitrieva et al. 2001). Although, the hypogastric nerve contains sympathetic and sensory neuropeptidergic fibers, the cervix contains few sympathetic fibers (Houdeau et al. 1998, Mowa \& Papka 2004). By contrast, a variety of peptide-containing fibers, including substance $P$ and vasoactive intestinal peptide are part of the hypogastric innervation of the cervix (Dalsgaard et al. 1983, Carvalho et al. 1986). The apparent absence of these projections following hypogastric nerve transection did not affect the processes of cervical remodeling or processes related to the normal timing of birth. All pups were born alive, on time, and without evidence of dystocia or forestalled labor. Activity by abdominal musculature to promote fetal expulsion (Higuchi et al. 1987) or smooth muscle 'ratcheting' to maintain intrauterine tension between contractions (Leppert 1995) may compensate for elimination of hypogastric innervation to ensure progression of labor and timely delivery of pups. Parturition could also progress without input from the hypogastric nerve if there was redundancy in neurotransmitter phenotypes innervating the cervix or if hypertrophy of nerve fibers from another nerve pathway compensates for loss of the hypogastric projection. For example, sympathetic fibers and projections from the paravertebral ganglia chain course with the splanchnic and pelvic nerves into the paracervical nerve plexus to innervate the cervix in NP rat (Hulsebosch \& Coggeshall 1982, Houdeau et al. 1995, Komisaruk et al. 1996). In the present study, thick bundles of nerve fibers were commonly present in the cervix by the day before birth in rats lacking hypogastric innervation, but were absent in sham-controls. Thus, changes in morphology and possibly activity of remaining nerve fibers may reinforce input from the $\mathrm{CN}$ to regulate processes associated with remodeling of the cervix and timing of birth.

In summary, sympathetic and peptidergic components of the hypogastric nerve do not appear to influence the process of cervical ripening or parturition. Absence of evidence for an alteration in collagen remodeling and immigration of macrophages into the prepartum cervix between rats following transection of the hypogastric nerve and sham controls suggest that cervical ripening progresses without hypogastric nerve innervation. Thus, the present study leads to the conclusion that other neural pathways contribute to the processes of cervical ripening and parturition.

\section{Materials and Methods}

NP and time-dated gravid Long Evans Rats were purchased from Harlan Sprague Dawley, Inc. (Indianapolis, IN, USA). Rats were housed singly on $12 \mathrm{~h}$ light: $12 \mathrm{~h}$ darkness cycle (lights off at $0700 \mathrm{~h}$ PST) with food and water ad libitum. Animals were cared for in accordance with National Institute of Health Office of Laboratory Animal Welfare policies and the protocol approved by the Institutional Animal Care and Use Committee.

\section{Experiment 1: time course study of peripartum cervical remodelling}

Rats that were NP or post-breeding day 15 (D15), day 19 (D19), day 21 (D21), day 21.5 (D21.5) or day 22 (post partum $\mathrm{PP}$, estimated as $4-8 \mathrm{~h}$ after birth) were killed with $\mathrm{CO}_{2}$ gas ( $n=4-5$ rats/group). The lower reproductive tract including the cervix, attached end of vagina, and body of uterus where horns converged was immediately removed and immersed in $4 \%$ paraformaldehyde. Tissue was obtained soon after lights on except for the day 21.5 group in which the procedure occurred a few hours before lights off. After $24 \mathrm{~h}$ the cervix was processed and embedded in paraffin. Cervices were sectioned longitudinally at $10 \mu \mathrm{m}$, mounted on positively charged glass slides, and processed as described below. Rats in this study served, in part, as unoperated controls to compare with sham-operated controls in Experiment 2.

\section{Experiment 2: effects of hypogastric nerve transection on cervical remodeling and birth}

Rats were anesthetized on day 14-15 post-breeding with an i.m. injection of ketamine $(90 \mathrm{mg} / \mathrm{kg})$ and xylazine $(10 \mathrm{mg} / \mathrm{kg})$. The protocol for transection of the hypogastric nerve followed previously described procedures (Cross \& Glover 1958, Cunningham et al. 1991, Cueva-Rolon et al. 1996, Dmitrieva et al. 2001). Briefly, a midline abdominal incision and blunt dissection exposed the descending aorta. The small bowel was gently retracted and wrapped in saline-saturated gauze. The hypogastric nerve was identified to course bilaterally on the surface of aorta caudal to the branch of the iliolumbar vessels and distal to the inferior mesenteric nerve plexus. Methylene blue dye was applied topically to clearly identify these myelinated nerve fibers (Seif et al. 2004). Rats were then randomly assigned to either a sham control group (Sham; $n=5$ ), in which the small bowel was replaced, or proceeded to undergo hypogastric neurectomy $(\mathrm{HnX} ; n=6)$. In the $\mathrm{HnX}$ group, the blue-tinged hypogastric nerves caudal to the inferior mesenteric ganglion were dissected free from fascia and the blood vessels and a bilateral $5 \mathrm{~mm}$ segment removed. In both 
groups, the abdominal musculature and fascia were closed with a running suture stitch and the skin secured with surgical staples. One pregnant rat with five implantation sites, two reabsorptions, and two full fetuses, in the right uterine horn and one fetus in the left horn, was excluded from the study. Post-operatively, yohimbine was administered to accelerate recovery from anesthesia $(10 \mathrm{mg} / \mathrm{kg}$, i.m). External compression of the bladder was performed periodically to express urine given the potential side effect of a weakened urethral sphincter reflex (Dmitrieva et al. 2001, Yoshiyama \& de Groat 2002). Near term, rats were observed at about $2-4 \mathrm{~h}$ intervals between $0800 \mathrm{~h}$ and $0700 \mathrm{~h}$. The cervix was obtained as described for rats in Experiment 1, above.

To confirm that this procedure abolished the hypogastric nerve projection to the cervix, NP and day 15 pregnant rats were subjected to the sham or hypogastrectomy procedure described above $(n=2-3$ Sham or $2 \mathrm{HnX}$ respectively for NP and pregnant groups). Before closing the abdomen, the cervix of each rat was injected in three separate locations with $4 \mu \mathrm{l}$ of $4 \%$ True Blue dye, a retrograde neural tract tracer. This method has been used to identify connections between the cervix and spinal cord (Berkley et al. 1993). After 7 days, rats were perfused with warm saline followed by $4 \%$ paraformaldehyde. The dorsal root ganglia in the thoracic (T10-T13) and lumbar (L1-L3) segments of the spinal cord was carefully excised and post-fixed overnight. Tissues were immersed in 30\% sucrose, cryostat sectioned at $30 \mu \mathrm{m}$, counterstained with mounting medium containing DAPI (Vector Labs, Burlingame, CA, USA), and fluorescence evaluated using an appropriate filter set (472-504 nm). True Blue fluorescence was only found in dorsal root ganglia from the lower thoracic segment of Sham rats (Fig. 5, pale grey-filled cells reflect green fluorescence). No fluorescent cells were present in dorsal root ganglia from thoracic segments of spinal cord in $\mathrm{HnX}$ rats or from segments of lumbar spinal cord in either group (data not shown). DAPI counterstain was present in all dorsal root ganglia (bright dots were seen as intense punctate violetblue fluorescence).

\section{Processing and staining of the cervix}

To visualize collagen structure and content, three $10 \mu \mathrm{m}$ crosssections of cervix from each rat were stained with picrosirius red as previously described (Leppert 1995, Yu et al. 1995, Luque et al. 1996, Kirby et al. 2005). A single black and white photograph, at $20 \times$ objective, was obtained from each section of cervix. Images were acquired in areas of cervix that did not contain luminal epithelium, uterus, vaginal tissue or other vacant spaces. In each image, nine adjacent but nonoverlapping grids were arranged in a cross pattern over the image. The optical density of polarized light in black and white photographs of each grid was analyzed by NIH Image J software with gray scale threshold calibrated using the Rodbard standard curve (http://rsb.info.nih.gov/ij/). Areas of high collagen content and complexity were bright red and, with conversion to black and white, became white regions of low optical density. By contrast, low collagen content and complexity were proportionally darker with higher optical density. Owing to the expected hypertrophy and hyperplasia of

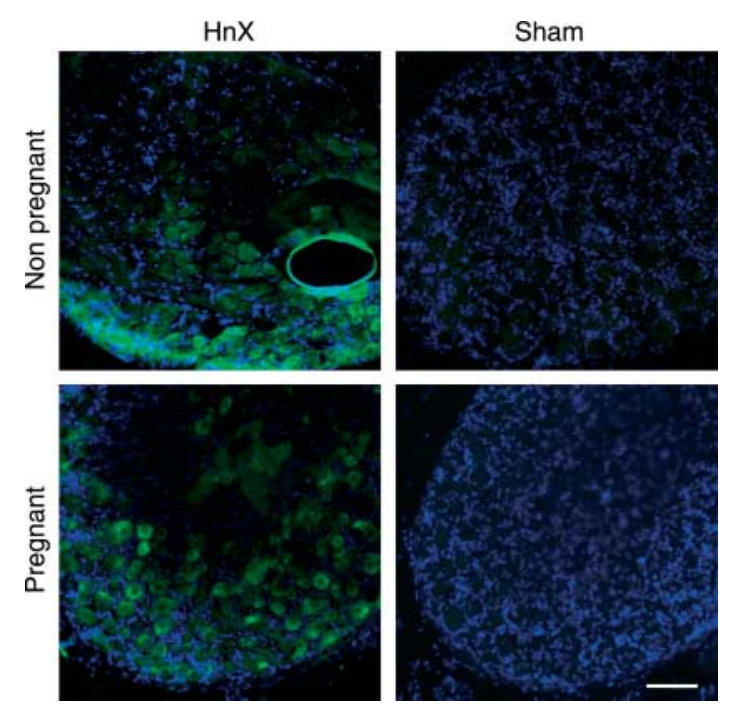

Figure 5 Photomicrographs of True Blue fluorescence in a dorsal root ganglion from the lower thoracic segment of the spinal cord in nonpregnant (top panels) or pregnant rats that were sham-operated (Sham) or hypogastric nerve transected $(\mathrm{HnX})$. Note individual and clustered green fluorescent-tagged cells along lower periphery of the dorsal root ganglion from Sham, but not $\mathrm{HnX}$ rats. DAPI counterstain is the bright blue-violet punctate fluorescence. True Blue was injected into the cervix of non-pregnant or day 15 pregnant rats after Sham or HnX surgery. Dorsal root ganglia were obtained 7 days later as described in detail in Materials and Methods. Scale bar $=100 \mu \mathrm{m}$.

the gravid versus non-gravid cervix, optical density data were normalized to the number of cell nuclei density for each rat. This method of analysis differs from the assessment of luminosity of collagen in the prepartum cervix by Marx et al. (2006) and allows direct comparisons with previous work in mice (Kirby et al. 2005, Yellon et al. 2008).

\section{Macrophage and nerve fiber immunohistochemistry}

Following previously described procedures (Mackler et al. 1999, Bosquiazzo et al. 2005, Kirby et al. 2005), slidemounted sections were treated with proteinase $\mathrm{K}$ for $3 \mathrm{~min}$, placed in a $3 \%$ hydrogen peroxide solution for $15 \mathrm{~min}$, then for 30 min each incubated with a blocking solution and then primary antisera ED-1 to visualize macrophages 1 1:200 dilution, Serotec, Oxford, UK). Biotinylated anti-mouse IgG was used as a secondary antibody (1:400 dilution; Vector Labs). For nerve fibers, sections were similarly processed except a $1 \%$ hydrogen peroxide solution was used and the primary antisera was to peripherin, a neurofilament IV-specific antigen (1:500 dilution, Novus Biologicals, Littleton, CO, USA). Biotinylated donkey anti-rabbit IgG served as the secondary antibody (1:200 dilution, Fitzgerald/RDI, Concord, MA, USA). In each run, incubation of sections without the primary antibody served as a negative control for non-specific stain. Macrophage counts and area with nerve fibers were normalized to cell nuclei density in each section to account for hypertrophy and hyperplasia of cervix among rats and with respect to pregnancy. 


\section{Statistical analyses}

Data were normally distributed $(P>0.05$, Levene's test for homogeneity of variance). Individual comparisons were made by ANOVA (Experiment 1) or with a Student's $t$-test (Experiment 2) using SPSS statistics software (Chicago, IL, USA). $P<0.05$ was considered statistically significant.

\section{Declaration of interest}

The authors declare that there is no conflict of interest that could be perceived as prejudicing the impartiality of the research reported.

\section{Funding}

Supported by NIH grant HD 054931, as well as by Richard Chinnock, MD, Chair, Department of Pediatrics, and H Roger Hadley, the Dean of Loma Linda University School of Medicine.

\section{Acknowledgements}

We thank Long Tran, John Chrisler, and Lindsey Vernon for technical expertise. The encouragement and support of Gerald and Susan Ebner, and Dennis and Franziska Shepard, has been greatly appreciated.

\section{References}

Baljet B \& Drukker J 1980 The extrinsic innervation of the pelvic organs in the female rat. Acta Anatomica 107 241-267.

Berkley KJ, Robbins A \& Sato Y 1993 Functional differences between afferent fibers in the hypogastric and pelvic nerves innervating female reproductive organs in the rat. Journal of Neurophysiology 69 533-544.

Bokstrom H, Brannstrom M, Alexandersson M \& Norstrom A 1997 Leukocyte subpopulations in the human uterine cervical stroma at early and term pregnancy. Human Reproduction 12 586-590.

Bosquiazzo VL, Durando M, Varayoud J, Ramos JG, Rodriguez HA, Munoz-De-Toro M \& Luque EH 2005 Macrophage density in the pregnant rat uterine cervix is modulated by mast cell degranulation. Journal of Reproductive Immunology 65 147-158.

Burden HW, Leonard M, Smith CP, Louis TM \& Lawrence IE 1984 The effects of pelvic neurectomy on collagen in the cervix of the pseudopregnant rat. Anatomical Record 210 575-581.

Burden HW, Price GT, Renegar RH \& Hodson CA 1990 Effects of peripheral nerve lesions during pregnancy on parturition in rats. Anatomy and Embryology 182 499-501.

Carlson RR \& De Feo VJ 1965 Role of the pelvic nerve vs. the abdominal sympathetic nerves in the reproductive function of the female rat. Endocrinology 77 1014-1022.

Carvalho TL, Hodson NP, Blank MA, Watson PF, Mulderry PK, Bishop AE, Gu J, Bloom SR \& Polak JM 1986 Occurrence, distribution and origin of peptide-containing nerves of guinea-pig and rat male genitalia and the effects of denervation on sperm characteristics. Journal of Anatomy 149 121-141.

Challis JRG, Sloboda DM, Alfaidy N, Lye SJ, Gibb W, Patel FA, Whittle WL \& Newnham JP 2002 Prostaglandins and mechanisms of preterm birth. Reproduction 124 1-17.

Collins PJ, Wilson K, \& Papka RE 1998 Secretoneurin-immunoreactive nerves of the female rat reproductive system. Neuroscience Meeting 28th 1618 .
Collins JJ, Lin CE, Berthoud HR \& Papka RE 1999 Vagal afferents from the uterus and cervix provide direct connections to the brainstem. Cell Tissue Research 295 43-54.

Cross BA \& Glover TD 1958 The hypothalamus and seminal emission. Journal of Endocrinology 16 385-395.

Cueva-Rolon R, Sansone G, Bianca R, Gomez LE, Beyer C, Whipple B \& Komisaruk BR 1996 Vagotomy blocks responses to vaginocervical stimulation after genitospinal neurectomy in rats. Physiology and Behavior 60 19-24.

Cunningham ST, Steinman JL, Whipple B, Mayer AD \& Komisaruk BR 1991 Differential roles of hypogastric and pelvic nerves in the analgesic and motoric effects of vaginocervical stimulation in rats. Brain Research $\mathbf{5 5 9}$ 337-343.

Dalsgaard CJ, Hokfelt T, Schultzberg M, Lundberg JM, Terenius L, Dockray GJ \& Goldstein M 1983 Origin of peptide-containing fibers in the inferior mesenteric ganglion of the guinea-pig: immunohistochemical studies with antisera to substance $P$, enkephalin, vasoactive intestinal polypeptide, cholecystokinin and bombesin. Neuroscience 9 191-211.

Dmitrieva N, Johnson OL \& Berkley KJ 2001 Bladder inflammation and hypogastric neurectomy influence uterine motility in the rat. Neuroscience Letters 313 49-52.

Doherty T \& Broide D 2007 Cytokines and growth factors in airway remodeling in asthma. Current Opinion in Immunology 19 676-680.

Ekman-Ordeberg G, Stjernholm Y, Wang H, Stygar D \& Sahlin L 2003 Endocrine regulation of cervical ripening in humans-potential roles for gonadal steroids and insulin-like growth factor-I. Steroids 68 837-847.

Facchinetti F, Venturini P, Blasi I \& Giannella L 2005 Changes in the cervical competence in preterm labour. BJOG : an International Journal of Obstetrics and Gynaecology 112 23-27.

Gintzler AR, Peters LC \& Komisaruk BR 1983 Attenuation of pregnancyinduced analgesia by hypogastric neurectomy in rats. Brain Research 277 186-188.

de Groat WC \& Theobald RJ 1976 Reflex activation of sympathetic pathways to vesical smooth muscle and parasympathetic ganglia by electrical stimulation of vesical afferents. Journal of Physiology 259 223-237.

Guevara-Guzman R, Buzo E, Larrazolo A, de la RC, Da Costa AP \& Kendrick KM 2001 Vaginocervical stimulation-induced release of classical neurotransmitters and nitric oxide in the nucleus of the solitary tract varies as a function of the oestrus cycle. Brain Research 898 303-313.

Hertelendy F \& Zakar T 2004 Prostaglandins and the myometrium and cervix. Prostaglandins, Leukotrienes and Essential Fatty Acids 70 207-222.

Higuchi T, Uchide K, Honda K \& Negoro H 1987 Pelvic neurectomy abolishes the fetus-expulsion reflex and induces dystocia in the rat. Experimental Neurology 96 443-455.

Holzer P 1988 Local effector functions of capsaicin-sensitive sensory nerve endings: involvement of tachykinins, calcitonin gene-related peptide and other neuropeptides. Neuroscience 24 739-768.

Houdeau E, Prud'homme MJ, Rousseau A \& Rousseau JP 1995 Distribution of noradrenergic neurons in the female rat pelvic plexus and involvement in the genital tract innervation. Journal of the Autonomic Nervous System 54 113-125.

Houdeau E, Rousseau A, Meusnier C, Prud'homme MJ \& Rousseau JP 1998 Sympathetic innervation of the upper and lower regions of the uterus and cervix in the rat have different origins and routes. Journal of Comparative Neurology 399 403-412.

Huber A, Hudelist G, Czerwenka K, Husslein P, Kubista E \& Singer CF 2005 Gene expression profiling of cervical tissue during physiological cervical effacement. Obstetrics and Gynecology 105 91-98.

Hulsebosch CE \& Coggeshall RE 1982 An analysis of the axon populations in the nerves to the pelvic viscera in the rat. Journal of Comparative Neurology 211 1-10.

Junqueira LC, Zugaib M, Montes GS, Toledo OM, Krisztan RM \& Shigihara KM 1980 Morphologic and histochemical evidence for the occurrence of collagenolysis and for the role of neutrophilic polymorphonuclear leukocytes during cervical dilation. American Journal of Obstetrics and Gynaecology 138 273-281.

Kirby LS, Kirby MA, Warren JW, Tran LT \& Yellon SM 2005 Increased innervation and ripening of the prepartum murine cervix. Journal of the Society for Gynecologic Investigation 12 578-585. 
Komisaruk BR, Bianca R, Sansone G, Gomez LE, Cueva-Rolon R, Beyer C \& Whipple B 1996 Brain-mediated responses to vaginocervical stimulation in spinal cord-transected rats: role of the vagus nerves. Brain Research 708 128-134.

Kong C, Gill BM, Rahimpour R, Xu L, Feldman RD, Xiao Q, McDonald TJ, Taupenot L, Mahata SK, Singh B et al. 1998 Secretoneurin and chemoattractant receptor interactions. Journal of Neuroimmunology $\mathbf{8 8}$ 91-98.

Lee JW \& Erskine MS 2000 Pseudorabies virus tracing of neural pathways between the uterine cervix and CNS: effects of survival time, estrogen treatment, rhizotomy, and pelvic nerve transection. Journal of Comparative Neurology 418 484-503.

Leppert PC 1995 Anatomy and physiology of cervical ripening. Clinical Obstetrics and Gynecology 38 267-279.

Longo LD \& Yellon S 1988 Biological timekeeping during pregnancy and the role of circadian rhythms in parturition. In The Endocrine Control of the Fetus, 173-191. Eds W Kunzel \& A Jensen. Berlin: Springer-Verlag.

Luque EH, Ramos JG, Rodriguez HA \& Munoz de Toro MM 1996 Dissociation in the control of cervical eosinophilic infiltration and collagenolysis at the end of pregnancy or after pseudopregnancy in ovariectomized steroid-treated rats. Biology of Reproduction $\mathbf{5 5}$ 1206-1212.

Mackler AM, lezza G, Akin MR, McMillan P \& Yellon SM 1999 Macrophage trafficking in the uterus and cervix precedes parturition in the mouse. Biology of Reproduction 61 879-883.

Martinez-Gomez M, Cruz Y, Pacheco P, Aguilar-Roblero R \& Hudson R 1998 The sensory but not muscular pelvic nerve branch is necessary for parturition in the rat. Physiology and Behavior 63 929-932.

Marx SG, Wentz MJ, Mackay LB, Schlembach D, Maul H, Fittkow C, Given R, Vedernikov Y, Saade GR \& Garfield RE 2006 Effects of progesterone on iNOS, COX-2, and collagen expression in the cervix. Journal of Histochemistry and Cytochemistry 54 623-639.

Melo RC \& Machado CR 1993 Noradrenergic and acetylcholinesterasepositive nerve fibres of the uterus in sexually immature and cycling rats. Histochemical Journal 25 213-218.

Mowa CN \& Papka RE 2004 The role of sensory neurons in cervical ripening: effects of estrogen and neuropeptides. Journal of Histochemistry and Cytochemistry 52 1249-1258.

Mowa CN, Usip S, Collins J, Storey-Workley M, Hargreaves KM \& Papka RE 2003 The effects of pregnancy and estrogen on the expression of calcitonin gene-related peptide (CGRP) in the uterine cervix, dorsal root ganglia and spinal cord. Peptides 24 1163-1174.

Osman I, Young A, Ledingham MA, Thomson AJ, Jordan F, Greer IA \& Norman JE 2003 Leukocyte density and pro-inflammatory cytokine expression in human fetal membranes, decidua, cervix and myometrium before and during labour at term. Molecular Human Reproduction 9 41-45.

Owman C 1981 Pregnancy induces degenerative and regenerative changes in the autonomic innervation of the female reproductive tract. Ciba Foundation Symposia 83 252-279.

Papka RE \& McNeill DL 1993 Light- and electron-microscopic study of synaptic connections in the paracervical ganglion of the female rat: special reference to calcitonin gene-related peptide-, galanin-and tachykinin (substance $\mathrm{P}$ and neurokinin $\mathrm{A}$ )-immunoreactive nerve fibers and terminals. Cell Tissue Research 271 417-428.

Papka RE \& Traurig HH 1988 Distribution of subgroups of neuropeptide Y-immunoreactive and noradrenergic nerves in the female rat uterine cervix. Cell Tissue Research 252 533-541.

Papka RE, Traurig HH \& Klenn P 1987 Paracervical ganglia of the female rat: histochemistry and immunohistochemistry of neurons, SIF cells, and nerve terminals. American Journal of Anatomy 179 243-257.

Peters LC, Kristal MB \& Komisaruk BR 1987 Sensory innervation of the external and internal genitalia of the female rat. Brain Research $\mathbf{4 0 8}$ 199-204.

Richardson JD \& Vasko MR 2002 Cellular mechanisms of neurogenic inflammation. Journal of Pharmacological and Experimental Therapeutics 302 839-845.
Sandner-Kiesling A, Pan HL, Chen SR, James RL, DeHaven-Hudkins DL, Dewan DM \& Eisenach JC 2002 Effect of kappa opioid agonists on visceral nociception induced by uterine cervical distension in rats. Pain 96 13-22.

Sato S, Hayashi RH \& Garfield RE 1989 Mechanical responses of the rat uterus, cervix, and bladder to stimulation of hypogastric and pelvic nerves in vivo. Biology of Reproduction 40 209-219.

Sato T, Ito A, Ogata Y, Nagase H \& Mori Y 1996 Tumor necrosis factor alpha $(\mathrm{TN}-\alpha)$ induces pro-matrix metalloproteinase 9 production in human uterine cervical fibroblasts but interleukin $1-\alpha$ antagonizes the inductive effect of TNF- $\alpha$. FEBS Letters 392 175-178.

Seif C, Martinez Portillo FJ, Osmonov DK, Bohler G, van der HC, Leissner J, Hohenfellner R, Juenemann KP \& Braun PM 2004 Methylene blue staining for nerve-sparing operative procedures: an animal model. Urology 63 1205-1208.

Steinman JL, Carlton SM \& Willis WD 1992 The segmental distribution of afferent fibers from the vaginal cervix and hypogastric nerve in rats. Brain Research 575 25-31.

Stjernquist M \& Owman C 1987 Interaction of noradrenaline, NPY and VIP with the neurogenic cholinergic response of the rat uterine cervix in vitro. Acta Physiologica Scandinavica 131 553-562.

Sullivan KA, Traurig HH \& Papka RE 1994 Ontogeny of neurotransmitter systems in the paracervical ganglion and uterine cervix of the rat. Anatomical Record 240 377-386.

Tanaka Y, Narahara H, Takai N, Yoshimatsu J, Anai T \& Miyakawa I 1998 Interleukin-1beta and interleukin-8 in cervicovaginal fluid during pregnancy. American Journal of Obstetrics and Gynaecology 179 644-649.

Temple JL, Bradshaw HB, Wood E \& Berkley KJ 1999 Effects of hypogastric neurectomy on escape responses to uterine distention in the rat. Pain $\mathbf{6}$ S13-S20.

Timmons BC \& Mahendroo MS 2006 Timing of neutrophil activation and expression of proinflammatory markers do not support a role for neutrophils in cervical ripening in the mouse. Biology of Reproduction 74 236-245.

Tornblom SA, Klimaviciute A, Bystrom B, Chromek M, Brauner A \& EkmanOrdeberg G 2005 Non-infected preterm parturition is related to increased concentrations of IL-6, IL- 8 and MCP-1 in human cervix. Reproduction Biology and Endocrinology 339.

Traurig HH, Papka RE \& Shew RL 1991 Substance P and related peptides associated with the afferent and autonomic innervation of the uterus. Annals of the New York Academy of Sciences 632 304-313.

Uchiyama T, Ito A, Ikesue A, Nakagawa H \& Mori Y 1992 Chemotactic factor in the pregnant rabbit uterine cervix. American Journal of Obstetrics and Gynaecology 167 1417-1422.

Vera PL, Haase EB \& Schramm LP 1997 Origins of the sympathetic innervation of the cervical end of the uterus in the rat. Brain Research $\mathbf{7 4 7}$ 140-143.

Yellon SM, Mackler AM \& Kirby MA 2003 The role of leukocyte traffic and activation in parturition. Journal of the Society for Gynecologic Investigation 10 323-338.

Yellon SM, Ebner CA \& Sugimoto Y 2008 Parturition and recruitment of macrophages in cervix of mice lacking the prostaglandin $\mathrm{F}$ receptor. Biology of Reproduction 78 438-444.

Yoshiyama M \& de Groat WC 2002 Effect of bilateral hypogastric nerve transection on voiding dysfunction in rats with spinal cord injury. Experimental Neurology 175 191-197.

Yu SY, Tozzi CA, Babiarz J \& Leppert PC 1995 Collagen changes in rat cervix in pregnancy - polarized light microscopic and electron microscopic studies. Proceedings of the Society for Experimental Biological Medicine 209 360-368.

Received 4 December 2008

First decision 20 January 2009

Accepted 20 January 2009 